syndrome: a common pathophysiological mechanism? Eur Neurol 1991; 31: 114-116.

7 Victor DI, Green WR, Stark WJ, Walsh FB. A non-permanent tonic pupil in rheumatoid arteritis. Can J Neurol Sci 1977; 4: 209-212.

8 Levy NS, Kramer SG, de Barros T. Pupillary and accommodative abnormalities in the Vogt-KoyanagiHarada syndrome. Am J Ophthalmol 1970; 69: 582-588.

9 Jean D, Maes E. Hyperthyroidism, ophthalmoplegia and unilateral mydriasis. Bull Soc Belge Ophthalmol 1992; 245: 29-32.

10 Stern BJ, Gruen R, Koeppel J, Aronson N, Krumholz A. Recurrent thyrotoxicosis and papilledema in a patient with communicating hydrocephalus. Arch Neurol 1984; 41: 65-67.

K Shikishima', K Kitahara' and K Inoue ${ }^{2}$

${ }^{1}$ Department of Ophthalmology, Jikei University

School of Medicine, Tokyo, Japan

${ }^{2}$ Department of Neurology, Jikei University School of Medicine, Tokyo, Japan

Correspondence: K Shikishima, Department of Ophthalmology, Jikei University School of Medicine, 3-25-8, Nishi-shinbashi, Minato-ku, Tokyo 105-8461, Japan

Tel: + 81334331111 ext. 3581;

Fax: +81334331936

E-mail: shiki@jikei.ac.jp

The authors have no commercial or proprietary interest in any material mentioned in this study. The authors also have received no financial support nor have any relationships with any entity with regard to the material presented in this study.

Part of this report was presented in July of 2004 at the 15th International Neuro-Ophthalmological Society Meeting in Geneva, Switzerland.

Eye (2006) 20, 613-615. doi:10.1038/sj.eye.6701937; published online 27 May 2005

Sir,

\section{Combined enucleation and orbitotomy for choroidal melanoma with orbital extension}

Extrascleral extension of uveal melanoma is observed in about $8 \%$ of cases and represents a poor prognostic factor. ${ }^{1,2}$ Management of orbital melanoma is controversial. In general, small nodular extrascleral extensions noted incidentally on ultrasonography are managed by modifying enucleation so as to avoid transection of the area of involvement. ${ }^{3}$ Beneficial role of adjuvant radiotherapy in such cases remains questionable. $^{3}$ Diffuse orbital involvement is best managed by exenteration, although long-term survival advantage offered by this procedure is not known. ${ }^{4-8}$ Larger but circumscribed orbital involvement may be excised via orbitotomy in conjunction with enucleation or proton beam radiotherapy.,10

We report a case of slow growing choroidal melanoma that led to a circumscribed intraconal orbital extension that was managed successfully by a combined approach of enucleation and orbitotomy.

\section{Case report}

A 76-year-old man presented with reduced vision of 3 years duration of the left eye in September, 1990. The past ocular history, personal, and family history was noncontributory. Visual acuity was $6 / 5$ in the right eye and $\mathrm{HM}$ in the left eye. The right eye was normal. Left eye had normal anterior segment. Fundus evaluation showed a diffuse melanocytic choroidal thickening estimated to be $9 \mathrm{~mm}$ in base and $3 \mathrm{~mm}$ in thickness in the macular region (Figure 1a and b). Associated overlying RPE changes and subtotal inferior retinal detachment was also present. A diffuse choroidal melanoma was diagnosed. Patient refused all forms of treatment but agreed for periodic observation. The lesion continued to grow slowly with development of extrascleral extension and iris neovascularization and neovascular glaucoma in February, 1999 (Figure 1c). As the eye was not painful, patient refused enucleation. In February 2003, there was onset of proptosis with enlargement of orbital component of the melanoma (Figure 1d). Systemic evaluation was negative for hepatic metastasis. Once the patient was made aware of the possibility of orbital exenteration in the near future, he agreed to undergo enucleation combined with orbitotomy so as to avoid disfigurement associated with exenteration.

The surgery was performed under general anaesthetic The initial surgical steps were of enucleation. Following disinsertion of all muscles, silk traction sutures were applied to the insertion of the recti muscles (Figure 1e). Superotemporal orbitomy was then performed through an upper eyelid crease incision (Figure 1f). Access to the intraconal orbit was gained by incising lateral orbital rim. The orbital melanoma could be palpated and it was isolated using blunt dissection. A long stump of optic nerve was cut and globe was removed with the orbital involvement. After haemostasis was achieved the orbitomy and enucleation wounds were closed with absorbable sutures (Figure 1g). There were no operative or postoperative complications. Ocular prosthesis was fitted 6 weeks postoperatively. Excellent cosmetic outcome could be achieved (Figure 1h). Patient continued to do well 1 year after the procedure. 

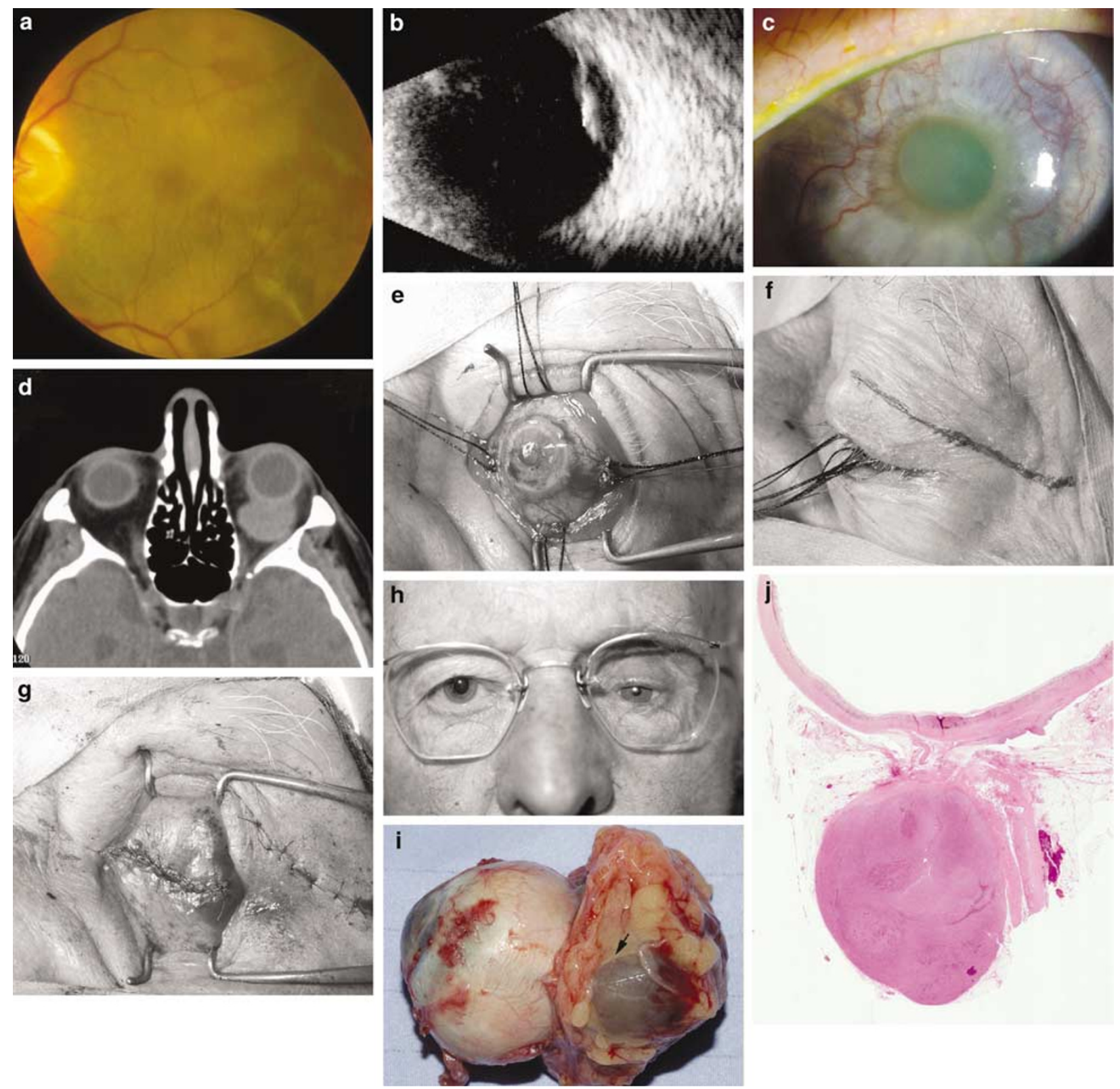

Figure 1 (a) Fundus photograph of the left eye at presentation showing a diffuse choroidal melanoma (1990). (b) There is choroidal thickening on B scan ultrasonograph. (c) The lesion continued to grow slowly with development of iris neovascularization and neovascular glaucoma (1999). (d) Circumscribed intraconal orbital involvement confirmed by axial CT scan. (e) The initial surgical steps were of enucleation. Following disinsertion of all muscles, silk traction sutures were applied to the insertion of the recti muscles. (f) Initiation of superotemporal orbitomy through an upper eyelid crease incision. (g) Appearance after closure of orbitomy and enucleation wounds. (h) Excellent cosmetic outcome at 6 months postoperatively. (i) Total excision of globe, optic nerve, and encapsulated orbital melanoma (arrow). (j) Diffuse choroidal melanoma with extensive posterior orbital component, abutting the optic nerve. (Haematoxylin and eosin, $\times 2$ ).

The excision of the orbital component was complete (Figure 1i). Histopathologic examination revealed an intraocular diffuse choroidal melanoma. This was of mixed cell type. The melanoma showed transcleral infiltration via the scleral canals with the presence of an extensive well-circumscribed posterior orbital component (Figure 1j). The latter showed orbital perineural invasion but did not invade the optic nerve tissues.

\section{Comments}

In our case, the orbital extension was circumscribed within the intraconal region of the orbit with sparing of 
the orbital apex. Although patient had been reluctant to undergo enucleation for several years, when possibility of impending exenteration was mentioned he readily agreed to undergo enucleation combined with orbitotomy. Simple enucleation would not have been possible without the risk of transecting the orbital component. Our technique avoided the exenteration with its consequent morbidity. Combined enucleation and orbitotomy should be considered for choroidal melanoma with circumscribed extension of melanoma into the orbit.

\section{References}

1 Anonymous . Histopathologic characteristics of uveal melanomas in eyes enucleated from the Collaborative Ocular Melanoma Study. COMS report no. 6. Am J Ophthalmol 1998; 125: 745-766.

2 Singh AD, Shields CL, Shields JA. Prognostic factors in uveal melanoma. Melanoma Res 2001; 11: 255-263.

3 Hykin PG, McCartney AC, Plowman PN, Hungerford JL. Postenucleation orbital radiotherapy for the treatment of malignant melanoma of the choroid with extrascleral extension. Br J Ophthalmol 1990; 74: 36-39.

4 Shammas HF, Blodi FC. Orbital extension of Choroidal and ciliary body melanomas. Arch Ophthalmol 1977; 95: 20022005.

5 Fenton S, Sandinha T, Lee WR, Kemp EG. Massive extraocular extension as the presenting feature of a choroidal melanoma. Eye 2001; 15: 550-551.

6 Kersten RC, Tse DT, Anderson RL, Blodi FC. The role of orbital exenteration in choroidal melanoma with extrascleral extension. Ophthalmology 1985; 92: 436-443.

7 Rini FJ, Jakobiec FA, Hornblass A, Beckerman BL, Anderson RL. The treatment of advanced choroidal melanoma with massive orbital extension. Am J Ophthalmol 1987; 104: 634-640.

8 Starr HJ, Zimmerman LE. Extrascleral extension and orbital recurrence of malignant melanomas of the choroid and ciliary body. Int Ophthalmol Clin 1962; 2: 369-385.

9 De Potter P, Shields JA, Shields CL, Santos R. Modified enucleation via lateral orbitotomy for choroidal melanoma with orbital extension: a report of two cases. Ophthal Plast Reconstr Surg 1992; 8: 109-113.

10 Weissgold DJ, Gragoudas ES, Green JP, Kent CJ, Rubin PA. Eye-sparing treatment of massive extrascleral extension of choroidal melanoma. Arch Ophthalmol 1998; 116: $531-533$

AD Singh'1, R Jacques², PA Rundle², HS Mudhar ${ }^{3}$ and IG Rennie ${ }^{2,4}$

${ }^{1}$ Department of Ophthalmic Oncology, Cole eye Institute, Cleveland Clinic Foundation (i3-129),

Cleveland, OH, USA

${ }^{2}$ Department of Ophthalmology, University of Sheffield, Sheffield, UK
${ }^{3}$ Department of Histopathology, University of

Sheffield, Sheffield, UK

${ }^{4}$ Department of Royal Hallamshire Hospital, University of Sheffield, Sheffield, UK

Correspondence: AD Singh,

Tel: + 216445 9479;

Fax: + 2164452226.

E-mail: SinghA@CCF.Org

Eye (2006) 20, 615-617. doi:10.1038/sj.eye.6701939; published online 17 June 2005

Sir,

\section{Subconjuntival cilia}

Eyelashes have been documented as growing in anomalous lid locations including meibomian gland orifices ${ }^{1}$ and burrowing beneath eyelid tissues - cilium incarnatum internum and externum. ${ }^{2}$ A paucity of literature exists regarding ectopic lashes, particularly conjunctival cilia. Scarring of the mobile bulbar and forniceal conjunctiva encourages the formation of blind pockets in which lashes can become trapped. ${ }^{3}$ We describe a case of cilia originating beneath the bulbar conjunctiva in a setting of previous intraocular surgery.

\section{Case report}

An 81-year-old lady attended outpatients in August 2004 describing recurrent foreign body sensation in the right eye. Past history included surgery for right total retinal detachment and bilateral YAG laser iridotomies for angle closure glaucoma. Best-corrected acuity was counting fingers right, 6/18 left. Slit-lamp examination revealed ectopic eyelashes originating from beneath the healed conjunctival peritomy made at the previous retinal surgery. There were around 13 lashes near the temporal limbus, approximately 10 had their bases inferiorly, extending superiorly, the remaining ones started superiorly and projected downwards. One lay horizontally (Figure 1). The overlying conjunctiva was mildly injected. Lid position and cornea appeared normal, and other than nuclear sclerotic cataract, there was no pathology of the left eye.

Hospital records revealed that 5 days prior to retinal surgery in 1995, the patient had fallen and preoperatively had periorbital ecchymoses. A single cilium beneath the bulbar conjunctiva was first observed in the patient's right eye in November 1996 and epilated. Following this, she presented at yearly intervals in September 2001, October 2002, and October 2003, with irritation of the 